\title{
Optimal Duration of Dual Antiplatelet Therapy After Two-Stent Treatment in Coronary Bifurcation Lesions
}

\author{
Yoshinobu Murasato, MD, $\mathrm{PhD}$
}

$\mathbf{I}$ n complex true coronary bifurcation lesions with severe or diffuse stenosis in both the main and side branches, drug-eluting stent (DES) deployment in both branches (2-stenting) is required. A systematic approach has been recently reported to provide less target lesion failure (TLF) than with the provisional approach. ${ }^{1}$ However, patients with high bleeding risk (HBR) are included in $>30 \%$ of this cohort of progressed atherosclerosis. The optimal period for continuation of dual antiplatelet therapy (DAPT) after 2-stenting has not yet been established.

In this issue of the Journal, Kang et al ${ }^{2}$ investigate 454 patients in the COBIS III registry who finally underwent 2-stenting for bifurcation lesions and report no difference in TLF according to stenting sequence (provisional or systematic) or technique. They also found that left main disease and short-term DAPT were associated with TLF, and the time period of the interaction of TLF and DAPT was as long as 2.5 years (Table). ${ }^{2}$ The efficacy of long-term DAPT is consistent with a previous pooled analysis of 6 randomized trials that demonstrated the efficacy of longterm DAPT on cardiac ischemic events (cardiac death, myocardial infarction [MI], and stent thrombosis [ST]) in complex percutaneous coronary intervention (PCI), including 2-stenting in bifurcation lesions (complex PCI, adjusted hazard ratio [HR], 0.56; 95\% confidence interval [CI], 0.35-0.89; noncomplex PCI, 1.01 [0.75-1.35], Pinteraction $=0.01) .{ }^{3}$ However, a pooled analysis of 8 trials,${ }^{4} 5$ of which were the same as in the previous study, ${ }^{3}$ was performed according to lesion complexity stratification and HBR (Table). The findings demonstrated that longterm DAPT significantly increased the bleeding risk in noncomplex PCI in HBR patients (absolute difference $+2.61 \%, P=0.003$ ), and a significant benefit on ischemic events was found only in non-HBR patients (complex PCI, $-3.86 \%, \mathrm{P}=0.04$; noncomplex PCI, $-1.14 \%, \mathrm{P}=0.05$ ), which indicated the inefficacy of long-term DAPT in complex PCI in HBR patients. ${ }^{4} \mathrm{~A}$ post-hoc analysis of EXCEL, one of the pivotal trials of unprotected left main disease including 2-stenting in $43 \%$, was performed according to the presence of DAPT continuation after 1 year, which presented a non-significant increase of primary endpoint risk (all-cause death, MI, stroke [HR, 1.59; 95\% CI, 0.69-3.48]) and death (2.69 [0.82-8.849]), which suggested the inefficacy of long-term DAPT. 5

\section{Article p 1944}

The effectiveness of 1-month DAPT after implantation of 2nd-generation DES with durable polymer ( $\mathrm{Pt}-\mathrm{Cr}$ everolimus-eluting stent, Co-Cr zotarolimus-eluting stent, and $\mathrm{Co}-\mathrm{Cr}$ everolimus-eluting stent) has been recently reported in all-comers' trials in HBR patients. ${ }^{68}$ In these studies, bifurcation lesions were enrolled in 15-25\% of cases. However, the 2-stenting ratio was either not described ${ }^{6,7}$ or extremely low. ${ }^{8}$ Hence, the safety of ultra-short-term DAPT after 2-stenting using these DES has not been confirmed. In a pooled analysis of 2 registries for left main and bifurcation lesions, CHANCE, using polymer-free biolimus-eluting stent (PF-BES) and in RAIN using 2nd-generation DES with a strut thickness of approximately $80 \mu \mathrm{m}$ (ultra-thin DES) (Table), 1-month DAPT after PCI using the ultra-thin DES resulted in more frequent ischemic events than when using PF-BES ( $22 \%$ vs. $12 \%, \mathrm{P}=0.04) .{ }^{9} \mathrm{~A}$ substudy of bifurcation lesions was conducted in the GLOBAL LEADERS using PF-BES with a randomized comparison between 1-month DAPT plus 23-month monotherapy of ticagrelor and 12-month conventional DAPT plus 12-month monotherapy of aspirin (Table). The substudy included a large number of 2-stenting cases (489 patients), which led to a higher composite endpoint of death and new-onset Q-MI than with the 1-stent treatment (7.2\% vs. $4.0 \%, \mathrm{P}=0.008$ ), and treatment with 1-month DAPT followed by monotherapy with ticagrelor resulted in less ST compared with conventional DAPT treatment (HR 0.46, 95\% CI 0.22-0.97). ${ }^{10}$ These findings indicate that using PFBES will likely result in rapid intimal coverage, and subsequent monotherapy with a potent antiplatelet agent is effective even in short-term DAPT after complex PCI in patients with HBR.

In the present study, ${ }^{2}$ probable and definite ST frequency was acceptably low (1.3\%) even after 2-stenting. However, short-term DAPT was identified as an independent risk factor for TLF, which suggested that TLF was related to subclinical thrombus attachment on the overlapped stent struts with delayed healing, malapposed struts, more floating struts at the branch ostium, and dissection at the stent edge of the gap between the 2 stents. There remains room for improvement in TLF before

The opinions expressed in this article are not necessarily those of the editors or of the Japanese Circulation Society.

Received May 13, 2021; accepted May 13, 2021; J-STAGE Advance Publication released online June 17, 2021

Department of Cardiology and Clinical Research Center, National Hospital Organization Kyushu Medical Center, Fukuoka, Japan

Mailing address: Yoshinobu Murasato, MD, PhD, Department of Cardiology, National Hospital Organization Kyushu Medical

Center, 1-8-1 Jigyohama, Chuo-ku, Fukuoka 810-8563, Japan. E-mail: y.murasato@gmail.com

All rights are reserved to the Japanese Circulation Society. For permissions, please e-mail: cj@j-circ.or.jp

ISSN-1346-9843 


\begin{tabular}{|c|c|c|c|c|c|c|}
\hline Study & Type & Date & Objective & Stent & $\begin{array}{l}\text { Bifurcation, } \mathbf{n} \\
\text { (\% to whole } \mathrm{PCl} \text { ) }\end{array}$ & $\begin{array}{l}\text { 2-stent, n (\% } \\
\text { to whole PCl) }\end{array}$ \\
\hline Giustino et $\mathrm{al}^{3}$ & $\begin{array}{l}\text { Pooled } 6 \text { RCTs } \\
\text { analysis* }\end{array}$ & 2016 & All comers & $\begin{array}{l}1 \text { st-generation } \\
\text { DES 12-15\%, } \\
\text { 2nd-generation } \\
\text { DES } 85-88 \%\end{array}$ & ND & $661(6.9)$ \\
\hline $\mathrm{EXCEL}^{5}$ & $\begin{array}{l}\text { Post-hoc analysis } \\
\text { in RCT }\end{array}$ & 2018 & UPLM & DP-EES & $633(100)$ & $272(43)$ \\
\hline SENIOR 6 & $\mathrm{RCT}$ & 2018 & $\begin{array}{l}\text { HBR (elderly, } \\
>75 \text { years }\end{array}$ & DP-EES vs. BMS & $263(14.9)$ & ND \\
\hline Costa et al ${ }^{4}$ & $\begin{array}{l}\text { Pooled } 8 \text { RCTs } \\
\text { analysis\#" }\end{array}$ & 2019 & All comers & $\begin{array}{l}\text { BMS 8-20\%, } \\
1 \text { st-generation } \\
\text { DES 6-10\%, } \\
\text { 2nd-generation } \\
\text { DES } 71-83 \%\end{array}$ & ND & $1,197(8.0)$ \\
\hline STOP-DAPT $2^{7}$ & RCT & 2019 & HBR & DP-EES & $769(25.6)$ & $16(0.5)$ \\
\hline CHANCE and RAIN ${ }^{9}$ & $\begin{array}{l}\text { Pooled } 2 \\
\text { registries analysis }\end{array}$ & 2020 & $\begin{array}{l}\text { UPLM and } \\
\text { bifurcation (CHANCE, } \\
\text { HBR } 77.7 \% \text { ) }\end{array}$ & $\begin{array}{l}\text { CHANCE: PF-BES, } \\
\text { RAIN: Ultra-thin DES }\end{array}$ & $843(100)$ & ND \\
\hline $\begin{array}{l}\text { GLOBAL LEADERS } \\
\text { Bifurcation }{ }^{10}\end{array}$ & $\mathrm{RCT}$ & 2020 & All comers & PF-BES & $2,498(100)$ & 489 (19.8) \\
\hline ONYX-ONE7 & $\mathrm{RCT}$ & 2020 & HBR & DP-ZES vs. PF-BES & $453(16.0)$ & ND \\
\hline COBIS III: 2-stent² & Registry & 2021 & UPLM and bifurcation & 2nd-generation DES & $454(100)$ & $454(100)$ \\
\hline
\end{tabular}

\begin{tabular}{|c|c|c|}
\hline Study & DAPT & Clinical results \\
\hline Giustino et $\mathrm{al}^{3}$ & $\begin{array}{l}\text { Short DAPT ( } 3-6 \text { months) } \\
\text { vs. Long DAPT } \\
\text { ( } \geq 12 \text { months) }\end{array}$ & $\begin{array}{l}\text { Long vs. short DAPT on primary endpoint (cardiac death, MI, ST), adjusted HR ( } 95 \% \\
\text { Cl): Complex PCI, } 0.56(0.35-0.89) \text {; Noncomplex PCI, } 1.01 \text { (0.75-1.35); Pinteraction=0.01. } \\
\text { Major bleeding risk: complex PCI, } 1.81 \text { (0.67-4.91); noncomplex PCI, } 1.75(0.98-3.12) \text {, } \\
\text { Pinterraction=0.96 }\end{array}$ \\
\hline $\mathrm{EXCEL}^{5}$ & $\begin{array}{l}\text { DAPT }<1 \text { year vs. } \\
\text { DAPT } \geq 1 \text { year }\end{array}$ & $\begin{array}{l}\text { Long vs. short DAPT on primary endpoint (Ml, death, stroke), HR } 1.59(95 \% \mathrm{Cl} \\
0.69-3.48 ; \mathrm{P}=0.28) \text {, all-cause death } 2.69(0.82-8.84, \mathrm{P}=0.10)\end{array}$ \\
\hline SENIOR ${ }^{6}$ & $\begin{array}{l}\text { DAPT } 1 \text { month for stable } \\
\text { AP, } 6 \text { months for ACS }\end{array}$ & $\begin{array}{l}\text { DP-EES vs. BMS on the primary endpoint (all-cause death, MI, stroke, TLR), relative } \\
\text { risk } 0.71(95 \% \mathrm{Cl} 0.52-0.94, \mathrm{P}=0.02)\end{array}$ \\
\hline Costa et $\mathrm{al}^{4}$ & $\begin{array}{l}\text { Short DAPT ( } 3-6 \text { months) } \\
\text { vs. long DAPT } \\
\text { ( } \geq 12 \text { months) }\end{array}$ & $\begin{array}{l}\text { Long vs. short DAPT, absolute difference: Ischemic events in non-HBR patients: } \\
\text { Complex } \mathrm{PCl},-3.86 \%, \mathrm{P}=0.04 ; \text { Noncomplex } \mathrm{PCl},-1.14 \%, \mathrm{P}=0.05 \text {. In HBR patients, } \\
\text { Complex } \mathrm{PCl},+1.30 \%, \mathrm{P}=0.76 \text {; Noncomplex } \mathrm{PCl},+1.45 \%, \mathrm{P}=0.39 \text {. Bleeding risk in } \\
\text { non-HBR patients: Complex } \mathrm{PCl},+0.28 \%, \mathrm{P}=0.57 ; \text { Noncomplex } \mathrm{PCl},+0.12 \%, \mathrm{P}=0.53 \text {. } \\
\text { In HBR patients, Complex } \mathrm{PCl},+3.04 \%, \mathrm{P}=0.30 \text {; Noncomplex } \mathrm{PCl}+2.61 \%, \mathrm{P}=0.003 \text {. }\end{array}$ \\
\hline STOP-DAPT $2^{7}$ & $\begin{array}{l}\text { 1-month DAPT vs. } \\
\text { 12-month DAPT }\end{array}$ & $\begin{array}{l}\text { Short vs. long DAPT in primary endpoint (cardiovascular death, MI, ST, stroke, major } \\
\text { bleeding), absolute difference, }-1.34 \%(-2.57 \% \text { to }-0.11 \%) ; \mathrm{HR}, 0.64(0.42-0.98) \text {, } \\
\text { noninferiority }(\mathrm{P}<0.001) \text {, superiority }(\mathrm{P}=0.04)\end{array}$ \\
\hline CHANCE and RAIN ${ }^{9}$ & $\begin{array}{l}\text { CHANCE: DAPT }<1 \text {-month } \\
40 \%,<12 \text {-months } 99.9 \% \text {. } \\
\text { RAIN: Operator's discretion }\end{array}$ & $\begin{array}{l}\text { Ultra-thin DES vs. PF-BES: primary endpoint (cardiac death, MI, TLR, ST) after } 1 \text {-year, } \\
9 \% \text { vs. } 8 \%, \mathrm{P}=0.56 ; 2 \text {-stent group, TLR } 8.7 \% \text { vs. } 4.5 \%, \mathrm{P}=0.75 \text {, ST } 4.3 \% \text { vs. } 3.2 \% \text {, } \\
\mathrm{P}=0.91 .1 \text {-month DAPT group, primary endpoint } 22 \% \text { vs. } 12 \%, \mathrm{P}=0.04\end{array}$ \\
\hline $\begin{array}{l}\text { GLOBAL LEADERS } \\
\text { Bifurcation }{ }^{10}\end{array}$ & $\begin{array}{l}\text { 1-month DAPT+23-month } \\
\text { ticagrelor vs. } 12 \text {-month } \\
\text { DAPT+12-month aspirin }\end{array}$ & $\begin{array}{l}\text { Primary endpoint (all-cause death, new Q-MI), 1-stent } 4.2 \% \text { vs. 2-stent } 7.0 \%, P=0.008 \text {. } \\
\text { Short vs. long DAPT on ST, HR 0.46, } 95 \% \text { Cl } 0.22-0.97\end{array}$ \\
\hline ONYX-ONE & 1-month DAPT & $\begin{array}{l}\text { DP-ZES vs. PF-BES, primary endpoint (cardiac death, MI, ST), risk difference, } 0.2 \% \text {, } \\
P=0.01 \text { for noninferiority; secondary endpoint (cardiac death, MI, TLR), risk differnce } \\
0.2 \%, P=0.007 \text { for noninferiority }\end{array}$ \\
\hline COBIS III: 2-stent² & Operator's discretion & $\begin{array}{l}\text { Risk of TLF: LM bifurcation, HR } 3.192(95 \% \mathrm{Cl} 1.568-6.500, \mathrm{P}=0.001) \text {; Short DAPT, } \\
0.988 / 1 \text {-month increase }(0.980-0.995, \mathrm{P}=0.002)\end{array}$ \\
\hline
\end{tabular}


encouragement of prolonged DAPT. (1) There is a dominance of systematic 2-stenting over provisional 2-stenting $(65.2 \%$ vs. $25.8 \%)$. In particular, a higher frequency of TLF after systematic 2-stenting (crush stenting used most frequently) in the non-left main bifurcation compared with provisional 2-stenting suggests priority of the provisional approach in this lesion. (2) Significant efficacy of intravascular ultrasound (IVUS) was not observed in this study. Although stent under-expansion in 2-stenting is a critical risk factor for TLF, and is documented well with IVUS, more detailed intravascular observation may be required. Optical coherence tomography with higher resolution and $3 \mathrm{D}$ reconstruction may be another option to improve clinical outcomes. ${ }^{11}$ (3) Efficacy of final kissing balloon inflation (FKB) after 2-stenting was not demonstrated. FKB is generally recommended to reduce TLF because of the wide stent expansion of both the main and side branches and the decrease in stent malapposition. ${ }^{12}$ Simulataneoulsy, FKB includes risks of non-uniform dilation, stent deformation, ${ }^{12}$ and remaining incomplete strut apposition after suboptimal guidewire recrossing. ${ }^{11}$ Performing assessments before and after FKB using intravascular imaging is appropriate.

The results of the present study of 2-stenting in bifurcation lesions raise the following clinical issues. First, a more detailed survey of thrombus-related failure during and after the procedure is necessary. Second, stricter selection of 2-stenting for HBR patients should be considered. If 2-stenting is inevitable, short-term DAPT followed by a potent antiplatelet agent is a potential alternative to longterm DAPT. Third, the priority of systematic 2-stenting over the provisional approach is limited in complex true bifurcation lesions, which is more evident in the non-left main bifurcation.

\section{Disclosure}

The author does not have any conflicts of interest.

\section{References}

1. Zhang JJ, Ye F, Xu K, Kan J, Tao L, Santoso T, et al. Multicentre, randomized comparison of two-stent and provisional stenting techniques in patients with complex coronary bifurcation lesions: The DEFINITION II trial. Eur Heart J 2020; 41: 2523-2536.

2. Kang J, Han JK, Yang HM, Park KW, Kang HJ, Gwon HC, et al. Comparison of 2-stenting strategies depending on sequence or technique for bifurcation lesions in the second-generation drugeluting stent era: Analysis from the COBIS (Coronary Bifurcation Stenting) III registry. Circ J 2021; 85: 1944-1955.

3. Giustino G, Chieffo A, Palmerini T, Valgimigli M, Feres F, Abizaid A, et al. Efficacy and safety of dual antiplatelet therapy after complex PCI. J Am Coll Cardiol 2016; 68: 1851-1864.

4. Costa F, Van Klaveren D, Feres F, James S, Räber L, Pilgrim T, et al. Dual antiplatelet therapy duration based on ischemic and bleeding risks after coronary stenting. J Am Coll Cardiol 2019; 73: $741-754$.

5. Brener SJ, Serruys PW, Morice MC, Mehran R, Kappetein AP, Sabik JF 3rd, et al. Optimal duration of dual antiplatelet therapy after left main coronary stenting. J Am Coll Cardiol 2018; 72: 2086-2087.

6. Varenne O, Cook S, Sideris G, Kedev S, Cuisset T, Carrié D, et al. Drug-eluting stents in elderly patients with coronary artery disease (SENIOR): A randomised single-blind trial. Lancet 2018; 391: $41-50$.

7. Windecker S, Latib A, Kedhi E, Kirtane AJ, Kandzari DE, Mehran R, et al. Polymer-based or polymer-free stents in patients at high bleeding risk. $N$ Engl J Med 2020; 382: 1208-1218.

8. Watanabe H, Domei T, Morimoto T, Natsuaki M, Shiomi H, Toyota T, et al. Effect of 1-month dual antiplatelet therapy followed by clopidogrel vs 12-month dual antiplatelet therapy on cardiovascular and bleeding events in patients receiving PCI: The STOPDAPT-2 randomized clinical trial. JAMA 2019; 321: $2414-2427$.

9. D’Ascenzo F, Gaido L, Bernardi A, Saglietto A, Franzé A, Ielasi A, et al. Safety and efficacy of polymer-free biolimus-eluting stents versus ultrathin stents in unprotected left main or coronary bifurcation: A propensity score analysis from the RAIN and CHANCE registries. Catheter Cardiovasc Interv 2020; 95: 522-529.

10. Kogame N, Chichareon P, De Wilder K, Takahashi K, Modolo $\mathrm{R}$, Chang CC, et al. Clinical relevance of ticagrelor monotherapy following 1-month dual antiplatelet therapy after bifurcation percutaneous coronary intervention: Insight from GLOBAL LEADERS trial. Catheter Cardiovasc Interv 2020; 96: 100111.

11. Okamura T, Nagoshi R, Fujimura T, Murasato Y, Yamawaki $\mathrm{M}$, Ono $\mathrm{S}$, et al. Impact of guidewire recrossing point into stent jailed side branch for optimal kissing balloon dilatation: Core lab 3D optical coherence tomography analysis. EuroIntervention 2018; 13: e1785-e1793.

12. Murasato Y, Finet G, Foin N. Final kissing balloon inflation: The whole story. EuroIntervention 2015; 11(Suppl V): V81 -V85. 